\title{
Über den Gehalt des Speichels an Rhodankalium bei Tuberkulösen.
}

\author{
Von \\ Dr. Zickgraf, \\ II. Arzt der Heilstätte.
}

Infolge einer Anregung ron Herrn Prof. Kobert habe ich an einer Reihe von Phthisikern der hiesigen Heilstätte für Lungenkranke Versuche über die Rhodanausscheidung im Speichel angestellt. Der Zweck der Untersuchung war der zu ergründen, ob sich im Verlaufe der Lungentuberkulose eine Verminderung beziehungsweise Aufhebung des Rhodangehaltes nachweisen lasse, wie dies z. B. von Edinger und Treupel ${ }^{1}$ ) in einzelnen Fällen gefunden wurde. Bekanntlich existiert über den Rhodangehalt des Speichels eine grosse Literatur. Die physiologischen und physiologisch-pathologischen Lehrbücher geben uns allerdings geringen Aufschluss, mag das von den vielen sich widersprechenden Angaben herrühren. Allgemein anerkannt aber dürfte sein, dass sich tatsächlich im Verlaufe verschiedener Krankheiten der Rhodangehalt des Speichels ändert, während manchen verschwindet. Nach Kobert ${ }^{2}$ ) fehlt bei harnsaurer Diathese das Rhodan häufig im Speichel, es rührt dies nach ihm daher, dass die Harnsäure unzersetzt ausgeschieden wird (Harnsäure und Kalilauge und Schwefel erbitzt gibt Rhodankali). Bei Diabetikern fand von Noorden $n^{3}$ in einigen Fällen ebenfalls kein Rhodan, Edinger und

1) Edinger und Treupel, Münchn. med. Wochenschr. 1901. Nr. 39. Daselbst ist auch J. A. Grober (Deutsch. Arch. f. klin. Med. 1901) zitiert, nach dem kachektische, dauernd schwer affizierte Kranke wenig oder gar kein Rhodanalkali ausscheiden sollen, 1. Phthisiker, 2. harnsaure Diathetiker.

2) Verhandlungen d. Kongresses f. innere Medizin (Disk.) 1902.

3) v. Noorden, Pathologie des Stoffwechsels. 
Treupel dagegen konnten das Fehlen von Rhodan nur bei Phthisikern konstatieren. $J$ ürgen $\mathbf{s}^{1}$ ) hat bei seinen umfangreichen und genauen Rhodanuntersuchungen, die hauptsächlich Ohrenerkrankungen betreffen, auch innere Krankheiten in den Bereich gezogen und einige recht beachtenswerte Tatsachen gefunden. Leider findet sich unter seinen Fällen kein Tuberkulöser. Es scheint aber auch nach diesen Untersuchungen das Erscheinen oder Feblen der Rhodan-Kaliumreaktion im Speichel mit einer gewissen Konstanz aufzutreten, und es wäre möglich an der Hand umfangreichen Materials daraus Schlüsse auf Stoffwechselvorgänge im erkrankten Organismus zu ziehen. Die Hoffnung auf irgend diagnostischen Wert der Rhodanreaktion bei inneren Krankheiten kann ich allerdings nicht teilen.

Von den rerschiedenen Untersuchungsmethoden auf Rhodan habe ich zwei ständig angewandt, die von Treviranus und die von Solera. Erstere besteht darin, dass Rhodansalze mit Eisenchlorid eine blutrote Farbe der Flüssigkeit verursachen, letztere beruht auf der Bildung von blauer Jodstärke, die Rhodansalze mit Jodsäure und Stärkekleister geben. Beide Proben, besonders die letztere, die ich der Einfachheit wegen vorzog, sind sehr empfindlich. Es enthält der Parotisspeichel nach Vierordta) $0,0091-0,0239 \%$ Rhodankalium. Die geringen Quantitäten, die zur Untersuchung meist genügen, einige Kubikmillimeter, enthalten demnach nur Spuren von Rhodan, und doch ist die Reaktion so prompt und durch den Farbenumschlag besonders bei der Probe von Soler a so scharf beweisend. So leicht in dieser Hinsicht die Untersuchung erscheint, so stösst sie doch auf einige recht erhebliche Schwierigkeiten. Vor allem muss man für jede der beiden Proben eine Reihe von Fehlerquellen ausschliessen. Es geben nämlich ausser verschiedenen anderen Säuren, auch Salizyl- und Salpetrige Säure, ferner eingespritztes oder eingenommenes Morphium oder Opium, nicht zuletzt auch Speisereste und zersetzter Mundschleim die gleichen Reaktionen. Vor dem letzteren schützt man sich am besten, wie ich es immer gemacht habe, durch gesonderte Entnahme des Parotisspeichels. Eine Hauptfehlerquelle bildet aber das Rauchen des Patienten. Der Tabaksrauch mit seinem Gehalt an Blausäure ist geeignet dem Speichel auf lange Zeit Rhodanreaktion zu verleihen. Jürgens hat uns darüber eine recht instruktive Mitteilung gegeben, und es ist möglich, dass viele widersprechende Angaben in der Literatur von diesem nicht vermiedenen Fehler herrühren. Jürgens fand

1) Jürgens, Über die diagnostische Bedeutung der Rhodanreaktion etc. Monatsschr. f. Ohrenheilk. 1904. Nr. 5.

2) Vierordt, Daten und Tabellen. 
nämlich noch am nächsten Tage nach dem Genuss einer einzigen $Z$ igarette Bläunng bei der Jodsäurereaktion. Erst am zweiten Tage verschwand die Reaktion. Ich habe bei meinen Untersuchungen, die an Heilstättenpatienten gemacht wurden, auf diesen Punkt besondere Rücksicht nehmen müssen. In der hiesigen Heilstätte werden die aufgenommenen Patienten erst auf eine Beobachtungsstation gelegt, und erst wenn sie durch viermalige tägliche Messung fieberfrei befunden wurden, dürfen sie spazieren gehen. Es ist ihnen also erst am vierten Tage Gelegenheit zu einer verbotenen Zigarre gegeben. Ich untersuchte am dritten Tage, es muss dann, selbst wenn die Kranken am letzten Tage ihrer Freibeit noch geraucht haben, nach $2^{1 / 2}$ Tagen jede Spur von einer durch Tabakrauch bedingten Blausäurereaktion verschwunden sein. Für die gesonderte Entnahme des Parotisspeichels benützte ich zuerst ein kleines Wattebäuschchen, das ich an den Ausführungsgang der Ohrspeicheldrüse andrückte. Dasselbe wurde dann mit Stärkekleisterlösung getränkt und etwas Jodsäurepulver ${ }^{1}$ ) aufgeschiittet. Es trat dann meist sofort die charakteristische Blaufärbung ein. Für die Probe von Treviranus benötigt man etwas mehr Fliissigkeit, es ist aber notwendig vor dem Zusetzen der verdünnten Eisenchloridlösung mit Salzsäure anzusäuern, um das Verwechseln mit anderen Säuren zu vermeiden. In vielen Fällen habe ich durch Katheterisieren des Ductus Stenonianus mit einem $8 \mathrm{~cm}$ langen Kapillarröhrchen mir das nötige Sekret verschafft, in manchen Fällen konnte man den Speichel in der hohlen Sonde herunterlaufen lassen, so reichlich war die Absonderung. Meist gelang es mir durch psychische Beeinflussung des Patienten -- Frage nach seinen Lieblingsspeisen oder Getränken - Speichelfluss zu erregen, in einigen Fällen aber konnte ich auf keine Weise überhaupt Sekret erhalten. Durch geduldiges mehrmaliges Katheterisieren aber stellte sich dann in allen Fällen Speichel ein, zum Teil sogar sehr reichlich. Das Katheterisieren ist in vielen Fällen leicht, indem die Mündung des Ductus Stenonianus in Form eines kleinen Hügels oder einer Schleimhautfalte kenntlich ist oder bei geöffnetem Munde $1-1^{1 / 2} \mathrm{~mm}$ breit klafft. Es ist, glaube ich, nötig, auf all diese Kleinigkeiten und Einzelheiten hinzuweisen. Ich habe selbst in manchen Fällen viel Zeit und Geduld nötig gebabt, um zum Ziele zu gelangen.

Über die Menge des ausgeschiedenen Parotisspeichels gibt Vierordt an, dass aus einer Drüse pro Stunde $2 \mathrm{~g}$, pro 24 Stunden aus beiden Drüsen $80-100 \mathrm{~g}$ ausgeschieden werden. Fubini erhielt durch Katheterismus in 30 Minuten 5,9 (2-12) cmm im Durch-

1) Acidum jodicum puriss. Merk. 
schnitt. Mir war es möglich, in einem Falle in fünf Minuten $0,421 \mathrm{~g}$ und bei einem anderen Patienten $0,390 \mathrm{~g}$ zu gewinnen. In einem Falle benutzte ich ein vorher gewogenes Paukenröhrchen zum Katheterisieren und hielt das Röhrchen während dieser kurzen Zeit selbst in die zufällig sehr weite Ausmündungsstelle. Es ist also sicher die ausgeschiedene Menge des Parotisspeichels eine äusserst verschiedene.

Im ganzen habe ich 60 Fälle untersucht. Von diesen gehörten dem I. Stadium nach Turban an 36, dem II. 16, dem III. 8. Die Diagnose Tuberkulose war bei 20 durch positiven Nachweis von Tuberkelbazillen im Sputum gesichert, bei den übrigen Kranken waren entweder Blutungen vorangegangen, oder die Diagnose durch den Befund und die Anamnese unzweifelhaft. Nur in einem Falle war es mir zweifelhaft, ob Tuberkulose vorlag. Ich habe absichtlich recht schwer erkrankte Patienten, darunter acht des dritten Stadiums, zu den Untersuchungen herangezogen, da Edinger und Treupel, ebenso Grober hervorheben, dass dauernd schwer affizierte, kachektische Kranken die Reaktion vermissen lassen. Dem entsprachen sieben von meinen Patienten, die als ungeeignet für Heilstättenbehandlung zum Teil bald entlassen wurden. Von diesen gaben fünf sofort charakteristische Reaktion. Zwei Kranke musste ich erst mehrmals katbeterisieren, bis ich das nötige Sekret bekam und bei einem Kranken war die Reaktion zwar bei mehreren Untersuchungen vorhanden, aber immer schwach. Bei den ibrigen Fällen habe ich stets starke und sofortige Bläuung bei der Jodsäurereaktion und raschen und deutlichen Farbenumschlag bei der Probe von Treviranus konstatiert. Nach meinen Untersuchungen, die sicher unter den nötigen Kautelen gemacht wurden, verändert also die Lungentuberkulose, auch in der schwersten Form den Rhodangehalt des Speichels nicht.

Dies Resultat ist aber noch aus einem anderen Grunde interessant. Der merkwürdige Gegensatz, der zwischen der Konstitution Tuberkulöser und harnsaurer Diathetiker herrscht, ist zweifelsolne durch eine verschiedene chemische Veranlagung des Organismus, die vielleicht schon in der Keimanlage vorhanden ist, bedingt. Bei der harnsauren Diathese ist aber durch Kobert und Grober das Fehlen des Rhodans im Speichel festgestellt, wenigstens in vielen Fällen. Es ist also das konstante Auftreten des Rhodans selbst bei schwer affizierten Lungenkranken ein neuer Beweis dafür, dass bei beiden Krankheiten verschiedenartige chemische Vorgänge im Körper vorhanden sind. Für die Skrofulose nun erwartet Prof. Czerny bei der Lösung der Frage nach der Disposition nur auf dem Wege der chemischen 
Untersuchungen weitere Fortschritte. Warum sollte dies mit gewisser Einschränkung nicht auch für die Tuberkulose gelten?

Was den Gehalt der übrigen Speicheldrüsen angeht, so sind die Ansichten über das Submaxillarissekret noch geteilt. Von der Sublingualis wird das Vorkommen von Rhodankali behauptet. Ich habe in 17 Fällen das Sekret beider Drüsen untersucht and immer beide Reaktionen angestellt. Das Resultat war stets positiv, wenn auch in zwei Fällen nur schwach. Auch unter diesen Patienten befanden sich drei sehr schwer Kranke des III. Stadiums, die aber deutliche Realition gaben. Im übrigen habe ich dieselben Vorsichtsmassregeln beobachtet wie bei der Untersuchung des Parotisspeichels. Um ein Einfliessen desselben zu vermeiden, verschloss ich beide Seiten mit einem Wattebausch und entnahm mit einem kleinen Wattebäuschchen das an der Caruncula sublingualis tropfenförmig hervorquellende Sekret. Es ist wohl kaum möglich unter normalen Verhältnissen das Sekret beider Drüsen zu trennen, da ihre Ausführungsgänge entweder nebeneinander oder zu einem einzigen vereinigt münden. Da aber beide Drüsen gleichen histologischen Bau haben, so darf der Schluss gezogen werden, dass beide auch gleiches Sekret liefern.

Bei den von mir untersuchten Fällen befanden sich auch mehrere Ohrenkranke. Es war mir interessant die Schlussfolgerungen J ü r gen s, die zum Teil auch von anderen Autoren bestätigt wurden, zu prüfen. Leider konnte ich in keinem einzigen Falle ein Versagen der Rhodanreaktion auf der kranken Seite finden. Da dies nur nach Zerstörung des Paukengeflechtes bezw. der Chorda tympani, also bei stärkeren Zerstörungen im Mittelohr eintritt, muss dies bei meinen Ohrenkranken ausgeschlossen werden. Es waren drei Kranke mit chronischem Ohrenfluss, bei denen fötide cholesteatomähnliche Massen beim Spülen entfernt wurden. Bei einem Patienten war vor einem Jahre die Radikaloperation gemacht, das Ohr aber noch nicht abgeheilt. Der Grund dafür war ein kleiner Sequester am Aditus ad antrum. Zum Schlusse danke ich Herrn Professor Kobert für die Anregung zu dieser Untersuchung und Herrn Dr. Billig für die Ermöglichung derselben. 